\title{
NUMERICAL EXPERIMENTS ON THE DECAY OF THREE-BODY SYSTEMS
}

\author{
M. J. Valtonen and S. J. Aarseth \\ Institute of Astronomy \\ University of Cambridge
}

\begin{abstract}
RESUMEN
Se prescntan resultados numéricos de cálculos de sistemas de tres cuerpos estelares. De entre los sistemas triples con separaciones iniciales análogas entre cada una de las estrellas, los sistemas de masas iguales son los más estables, con vidas medias típicas de aproximadamentc 40 tiempos de cruce. En el otro extremo, sistemas con muy pequeño momento angular total o bien con una gran diferencia de masa, tienen vidas medias de sólo 10 tiempos de cruce aproximadamente. La cstrella que escapa es normalmente la menos masiva, mientras que la probabilidad de escape para la más "pesada" tiende a cero cuando su masa excede la masa combinada de las otras dos estrellas por un factor de 5. La binaria resultante se hace muy excéntrica $(\overline{\mathrm{e}} \geq 0.8)$ cuando las tres estrellas están restringidas a un plano y no poseen un momento angular total muy grande, o bien cuando el momento angular total es muy pequeño.
\end{abstract}

\begin{abstract}
Numerical results are presented for calculations of stellar three-body systems. Among the triple systems with similar initial separations between each of the stars, the equal-mass systems are most stable with typical half-lives of about 40 crossing times. In the other extreme, systems with a very small total angular momentum or a large mass difference have half-lives of only about 10 crossing times. The escaping star is usually the lightest one, while the escape probability for the heaviest star approaches zero when its mass exceeds the combined mass of the other two stars by more than a factor of about 5 . The final binary becomes very eccentric $\left(e_{f} \geq 0.8\right)$ when the three stars are restricted to a plane and do not have a very high total angular momentum, or when the total angular momentum is very small.
\end{abstract}

\section{INTRODUGTION}

The presence of small groups of stars in the Galaxy poses a well-defined dynamical problem which can be explored by numerical calculations. In general such systems are unstable; their members are lost to the surrounding field mainly by close encounters. If we assume no significant mass loss by internal stellar evolution, energy conservation implies that the final system should consist of at least one binary. This mechanism of binary formation is by no means unique, and in any case the total number of binarics is too great to have been produced in this way alone. However, a dynamical origin does have the merit of simplicity and this feature encourages the study of small stellar systems.

We restrict ourselves here to the simple case of three interacting particles with two of the particles already forming a binary. This procedure does not necessarily imply that the final binary configuration is pre-determined. In any case a more general threebody system cvolves to this type of configuration rather quickly. Alternatively, the initial system may be considered to have consisted of more particles which escaped, leaving one bound pair among the remaining three members (cf. van Albada 1968).

In the present investigation we adopt the method of statistical sampling for the appropriate secondary 
parameters. Previous analysis of these calculations (Valtonen 1974) indicates that already 200-300 combined examples provide a satisfactory statistical description for each specific choice of primary parameters (masses and energies). Such a collection of data constitutes a set of experiments; other sets are then obtained by considering different primary parameters. The earlier analysis also concluded that numerical errors which are present do not introduce a systematic bias in the final distributions.

\section{INITIAL CONDITIONS AND NUMERICAL TEGHNIQUE}

A complete description of the initial conditions for the three-body experiments is given elsewhere (Saslaw, Valtonen and Aarseth 1974; hereafter referred to as SVA). Initially the system is composed of two parts: a binary with component masses $\mathrm{m}_{1}$ and $\mathrm{m}_{2}$, semimajor axis $a$ and eccentricity $\mathrm{e}$; and $a$ third particle of mass $m_{3}$ in an elliptic or hyperbolic orbit relative to the binary centre of mass. The semi-major axis of the outer orbit is denoted by $a_{3}$ and its inclination relative to the binary plane by i. If $\left|a_{3}\right|>2020$ a, the integration stars with $\mathrm{m}_{3}$ approaching at a distance of $20 \mathrm{a}$ from the binary centre of mass, otherwise the initial position of $m_{3}$ is taken to be the apocentre point of the outer orbit.

The integration method makes use of a fourthorder fitting polynomial for the accelerations (Aarseth 1971) and the closest two-body encounter at any time is treated by a regularization technique (Heggie 1973). Scaled units are chosen such that the gravitational constant is unity. In addition we adopt $m_{1}+m_{2}=1$ (note the convention $m_{1} \geq m_{2}$ ) with a corresponding initial semi-major axis $a=1$, making the binary period equal to $2 \pi$ time units. The particle orbits are integrated for a maximum interval of $2000 \pi$ time units in general or 670 units in strongly bound systems $\left(a_{3} \sim 2\right)$, or until one of the particles satisfies the asymptotic escape criterion $a_{3}<0$.

In all experiments we randomize the mean anomaly of the binary as well as the ascending node and the pericentre length of the outer orbit. The masses have either fixed values within each set or are randomly distributed between certain limits (see SVA). The relative orientations of the binary and the outer orbit are usually random, but sometimes the orbits are in one plane $\left(i=0^{\circ}\right.$ or $\left.180^{\circ}\right)$. The binary has either zero eccentricity $(e=0)$ or a combination of zero and higher eccentricities (e $\sim$ 0.5 ; see SVA).

\section{LIFETIMES}

Figure 1 shows the frequency distribution of the lifetime $\mathrm{T}$ of the triple systems in 16 different sets of experiments. The lifetimes are measured in units of the crossing time, $T_{c r}=M^{5 / 2}|2 E|^{-3 / 2}$, where $M$ and $\mathrm{E}$ denote the total mass and energy of the triple

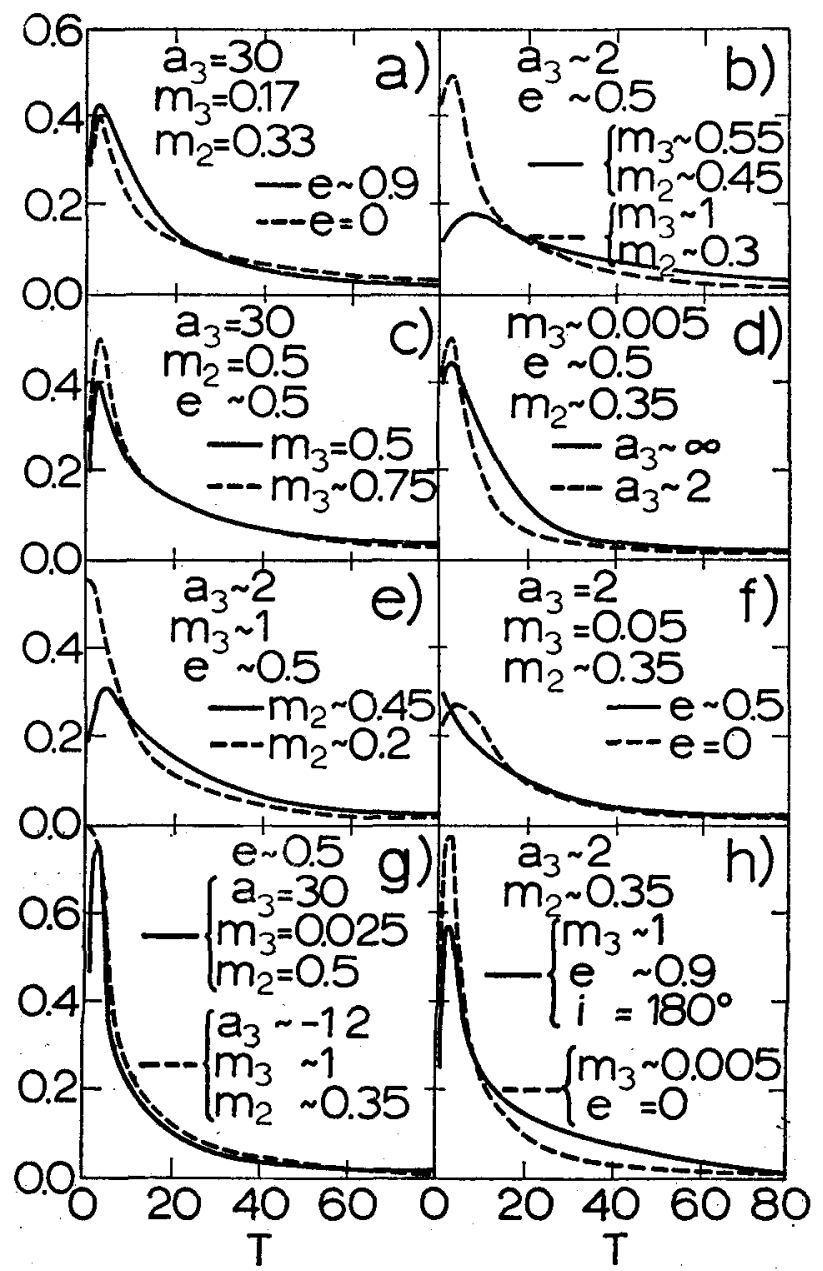

FIG. 1. The frequency distribution of life times of triple systems. 
system, respectively. The curves are shown for the first 80 crossing times when more than half and frequently more than three-quarters of all the systems have been disrupted. The main parameters of each set of experiments are given in the insert. In the sets where $a_{3} \gg 2$ or $a_{3}<0$, the ordinate is normalized relative to the total number of systems with non-zero lifetime. The lifetime is asumed to be zero if a system becomes unbound as a result of the first encounter between the binary and the third particle. In the other sets the normalization is carried out with respect to all experiments.

It is a characteristic feature of diagrams $(b),(c)$ and $(e)$ that the lifetime tends to increase as the mass dispersion is reduced. Here the solid lines refer to systems with a smaller mass range than the systems represented by dashed lines. Compare also the solid line in diagram $(c)$ with the solid line in diagram $(g)$, and the dashed line in diagram $(b)$ with the solid line in diagram $(f)$. Note that the latter set contains a relatively large number of zero lifetime systems. The apparent difference between the dashed lines in diagrams $(f)$ and $(h)$ may be due to the small number of experiments (120) in the former set; i.e., generally the distributions are based on 200 700 examples.

The effect of changing the total angular momentum can be seen from the distributions in diagram (a). Thus systems with a greater total angular momentum (dashed line) are more stable. The similarity of the dashed line in diagram $(b)$ and the solid line in diagram $(h)$ shows, on the other hand, that it is the total angular momentum rather than the inclination which is the more important parameter.

The effect of the total energy can be evaluated by comparing the dashed line in diagram $(b)$ with the dashed line in diagram $(g)$, or by comparing the two lines in diagram $(d)$ with each other. The differences in lifetimes do not appear to be significant. However, the difference between the solid lines in diagrams $(b)$ and $(c)$ indicates that strongly bound systems are more stable than weakly bound ones, at least when the masses are nearly equal. The same trend also appears for the solid lines in diagrams $(f)$ and $(g)$.

The typical half-lives of strongly bound systems of equal mass are $\simeq 40$ crossing times, whereas in weakly bound or low angular momentum systems, or in systems with a large mass difference, the half-life becomes as small as 10 crossing times (Valtonen 1974). Other calculations of this type (i.e., mostly in two dimensions) give similar results (cf. Agekyan and Anosova 1968). It is also interesting to note that the half-lives of three-body systems are in qualitative agreement with the harmonic mean escape times obtained for $\mathrm{N}$-body systems of 10 particles with different mass dispersions (van Albada 1968).

\section{ESCAPE PROBABILITIES}

Three-body systems usually desintegrate by forming a binary composed of the two heavier bodies and ejecting the lightest particle. For example, the probability of escape of a particle with mass 1.0-1.5 times greater than the combined mass of the other two particles is only a few per cent. When the mass difference becomes even greater, the escape probability for the heavy particle diminishes even further and approaches zero for a corresponding mass ratio of about 5 .

In general, the probability of escape does not depend on the initial conditions once the values of the masses have been fixed. Only the total angular momentum of a triple system has an effect when it is very small or very large. The ejection of a heavy mass becomes less likely in a very low angular momentum system, whereas in the high angular momentum case it is proportionately more likely. (SVA; Valtonen 1975).

\section{BINARY ECGENTRIGITIES}

Most distributions of the final binary eccentricities, $\mathbf{e}_{\mathfrak{f}}$, are in good agreement with theoretical considerations based on statistical equilibrium which give $f\left(e_{t}\right)=2 e_{t}$ and $\bar{e}_{t}=2 / 3$ (Heggie 1975). The main deviation from this distribution occurs in planar systems, where $\bar{e}_{f} \geq 0.8$. As the mass range is increased, the distribution tends to become flatter (Valtonen 1976). We also note that a very small total angular momentum causes an excess of eccentric binaries (Valtonen 1974). On the other hand, planar systems with large angular momenta produce an 
eccentricity distribution which is not very different from the theoretical prediction (SVA).

E

\section{DISGUSSION}

Although the present three-body experiments consist of a large number of examples, the initial conditions were not specifically selected to simulate multiple star systems. Nevertheless, the cases when the motion of the third particle is elliptic with respect to the binary may also be relevant here and most of the adopted mass ratios should apply. Not much is known about the total angular momentum of real systems. It is significant, however, that there is a strong tendency for the stars to be arranged in hierarchical configurations. This feature can be readily understood in terms of stability considerations; i.e., the lifetimes are relatively short if the separations are comparable, whereas systems with uneven spacings can be very stable (Harrington 1972). In the absence of observational data on the velocities, we may therefore assume that the outermost stars in old multiple systems have high angular momenta and thereby avoid having close encounters.

Another characteristic of the multiple systems (as indeed of many field stars) is that very close binaries are fairly common. Such close pairs have essentially the same dynamical effect as single stars of the corresponding mass, hence moclel calculations based on the three-body problem (say) may well be applicable to systems containing more members. For dynamical purposes it is useful to distinguish between interacting and non-interacting binaries, where the latter may be assumed to have approximately unperturbed relative motions for the times of interest. This approximation of a realistic simulation would reduce the computational requirements and permit more systems to be studied. It may also be noted that binaries with small dimensions compared to the size of the system are unlikely to have been formed dynamically, unless the remaining stars constitute a small proportion of the initial population.

Stability considerations involving real systems require an appropriate scaling of the dimensionless numerical results in order to introduce the physical time. The present calculations refer to isolated systems, hence applications should be restricted to parameters for which the effect of passing clouds and stars can be negiected. Expressing the mean harmonic radius, $R$, in $A U$ and the total mass in solar masses gives a crossing time in years of $t_{c r} \simeq 0.46\left(R^{3} / M\right)^{1 / 2}$. The lifetimes of nearly all the calculated triple systems would therefore be significantly shorter than the average age of low-mass stars for most lengthscales of interest, i.e., $R \leq 10^{4}$. This implies that multiple systems require especially favourable initial conditions in order to retain their members over long times.

One of the authors (M. J. V.) was supported by an Osk. Huttusen Säätiö Foundation Fellowship during this work.

\section{REFERENCES}

Aarseth, S. J. 1971, Ap. and Space Sci., 14, 118.

Agekyan, T. A., and Anosova, Z. P. 1968, Astrofizika, 4, 11.

Harrington, R. S. 1972, Celes. Mech., 6, 322.

Heggie, D. C. 1973, in Recent Advances in Dynamical Astronomy, eds. B. D. Tapley and V. Szebehely, (Dordrecht: D. Reidel), 34.

Heggie, D. C. 1975, M.N.R.A.S., 173, 729.

Saslaw, W. C., Valtonen, M. J., and Aarseth, S. J. 1974, Ap. J., 190, 253.

Valtonen, M. J. 1974, Proc. IAU Symposium No. 62, The Stability of the Solar System and of Small Stellar Systems, ed. Y. Kozai (Dordrecht: D. Reidel), 211.

Valtonen, M. J. 1975, Mem. R.A.S., 80, 77.

Valtonen, M. J. 1976 , to be published.

van Albada, T. S. 1968, Bull. Astr. Inst. Netherl., 19, 479.

\section{DISCUSSION}

Abt: If this is true, there should be very few triple systems which are older than roughly 100 times the long period. But I would suggest that there are many such violations.

King: I am sure that the answer to Abt's question is that your cases differ from classical triples in that you have aimed the third star almost directly at the binary. How closely did you aim?

Aarseth: The impact parameters were distributed within about twice the semi-major axis of the binary. 